\title{
MONTE CARLO TRACKING ON THE RIEMANNIAN MANIFOLD OF MULTIVARIATE NORMAL DISTRIBUTIONS.
}

\author{
Hichem Snoussi* and Cédric Richard \\ Charles Delaunay Institute, \\ FRE CNRS 2848, University of Technology of Troyes, France \\ Email=Hichem.Snoussi@utt.fr
}

\begin{abstract}
In this contribution, a general scheme of particle filtering on Riemannian manifolds is proposed. In addition to the nonlinear dynamics, the system state is constrained to lie on a Riemannian manifold $\mathcal{M}$, which dimension is much lower than the whole embedding space dimension. The Riemannian manifold formulation of the state space model avoids the curse of dimensionality from which suffers most of the particle filter methods. Furthermore, this formulation is the only natural tool when the embedding Euclidean space cannot be defined (the state space is defined in an abstract geometric way) or when the constraints are not easily handled (space of positive definite matrices).
\end{abstract}

\section{INTRODUCTION}

Given a dynamical system characterized by a state-space model, the objective of the online Bayesian filtering is the estimation of the posterior marginal probability of the hidden state given all the observations collected until the current time. The nonlinear and/or the non Gaussian aspect of the prior transition distributions and the observation model leads to intractable integrals when evaluating the marginals. Therefore, one has to resort to approximate Monte Carlo schemes. Particle filtering [1] is such an approximate Monte Carlo method estimating, recursively in time, the marginal posterior distribution of the continuous hidden state of the system. The particle filter provides a point mass approximation of these distributions by drawing particles according to a proposal distribution and then weighting the particles in order to fit the target distribution.

The particle filter method is usually applied to track a hidden state belonging to an Euclidean space. The most popular scheme is to sample the particles according to a random walk around the previous particles. However, in some tracking applications, the state may be constrained to belong to a Riemannian manifold. Recently, some works have been dedicated to design algorithms adapted to the Riemannian manifold constraints, based on differential geometry tools: Gradient-descent algorithm on Grassmann manifold for object recognition in [2], statistical analysis of diffusion tensor MRI in [3], geodesic-based deconvolution algorithms in [4], tracking principal subspaces in [5] and a general scheme for tracking fast-varying states on Riemannian manifolds in [6]. This paper contribution is devoted to the application of this differential-geometric framework to design efficient target tracking algorithms. We particularly consider the case where the observation noise covariance is unknown and time-varying. The Bayesian filtering objective is thus to jointly estimate the hidden target state and the time-varying noise covariance. As the noise covariance is a positive definite matrix, the Euclidean space is not suitable when tracking this covariance. Instead, one should exploit the differential geometric properties of the space of positive definite matrices, by constraining the estimated matrix to move along the geodesics of this Riemannian manifold. The proposed sequential Bayesian updating consists thus in drawing state samples while moving on the manifold geodesics.

The paper is organized as follows: Section 2 is a brief introduction to the particle filtering method on the Euclidean spaces. In Section 3, we describe some concepts of differential geometry. In Section 4, we present a general scheme for the particle filtering method on a Riemannian manifold. Section 5 is dedicated to the main contribution of this paper which is the design of a particle filter jointly tracking a target state belonging to an Euclidean space and a timevarying noise covariance modeling the evolution over time of the sensing system imperfections.

\section{BAYESIAN FILTERING ON EUCLIDEAN SPACES}

The observed system evolves in time according to the following nonlinear dynamics:

$$
\left\{\begin{aligned}
\boldsymbol{x}_{t} & \sim p_{x}\left(\boldsymbol{x}_{t} \mid \boldsymbol{x}_{t-1}, u_{t}\right) \\
\boldsymbol{y}_{t} & \sim p_{y}\left(\boldsymbol{y}_{t} \mid \boldsymbol{x}_{t}, u_{t}\right)
\end{aligned}\right.
$$


where $\boldsymbol{y}_{t} \in \mathbb{R}^{n_{y}}$ denotes the observation at time $t, \boldsymbol{x}_{t} \in$ $\mathbb{R}^{n_{x}}$ denotes the unknown continuous state, and $u_{t} \in \mathcal{U}$ denotes a known control signal. The probability distribution $p_{x}\left(\boldsymbol{x}_{t} \mid \boldsymbol{x}_{t-1}, u_{t}\right)$ models the stochastic transition dynamics of the hidden state. Given the continuous state, the observations $\boldsymbol{y}_{t}$ follow a stochastic model $p_{y}\left(\boldsymbol{y}_{t} \mid \boldsymbol{x}_{t}, u_{t}\right)$, where the stochastic aspect reflects the observation noise.

The Bayesian filtering is based on the estimation of the posterior marginal probability $p\left(\boldsymbol{x}_{t} \mid \boldsymbol{y}_{1: t}\right)$. Because of the nonlinear and/or the non Gaussian aspect of the transition distributions, one has to resort to Monte Carlo approximation where the joint posterior distribution $p\left(\boldsymbol{x}_{0: t} \mid \boldsymbol{y}_{1: t}\right)$ is approximated by the point-mass distribution of a set of weighted samples (called particles) $\left\{\boldsymbol{x}_{0: t}^{(i)}, w_{t}^{(i)}\right\}_{i=1}^{N}$ :

$$
\hat{p}_{N}\left(\boldsymbol{x}_{0: t} \mid \boldsymbol{y}_{1: t}\right)=\sum_{i=1}^{N} w_{t}^{(i)} \delta_{\boldsymbol{x}_{0: t}^{(i)}}\left(d \boldsymbol{x}_{0: t}\right),
$$

where $\delta_{\boldsymbol{x}_{0: t}^{(i)}}\left(d \boldsymbol{x}_{0: t}\right)$ denotes the Dirac function.

In the Bayesian importance sampling (IS) method, the particles $\left\{\boldsymbol{x}_{0: t}^{(i)}\right\}_{i=1}^{N}$ are sampled according to a proposal distribution $\pi\left(\boldsymbol{x}_{0: t} \mid \boldsymbol{y}_{1: t}\right)$ and $\left\{w_{t}^{(i)}\right\}$ are the corresponding normalized importance weights:

$$
w_{t}^{(i)} \propto \frac{p\left(\boldsymbol{y}_{1: t} \mid \boldsymbol{x}_{0: t}^{(i)}\right) p\left(\boldsymbol{x}_{0: t}^{(i)}\right)}{\pi\left(\boldsymbol{x}_{0: t}^{(i)} \mid \boldsymbol{y}_{1: t}\right)}
$$

Sequential Monte Carlo (SMC) consists of propagating the trajectories $\left\{\boldsymbol{x}_{0: t}^{(i)}\right\}_{i=1}^{N}$ in time without modifying the past simulated particles. This is possible for the class of proposal distributions having the following form:

$$
\pi\left(\boldsymbol{x}_{0: t} \mid \boldsymbol{y}_{1: t}\right)=\pi\left(\boldsymbol{x}_{0: t-1} \mid \boldsymbol{y}_{1: t-1}\right) \pi\left(\boldsymbol{x}_{t} \mid \boldsymbol{x}_{0: t-1}, \boldsymbol{y}_{1: t}\right) .
$$

The normalized importance weights are then recursively computed in time as:

$$
w_{t}^{(i)} \propto w_{t-1}^{(i)} \frac{p\left(\boldsymbol{y}_{t} \mid \boldsymbol{x}_{t}^{(i)}\right) p\left(\boldsymbol{x}_{t}^{(i)} \mid \boldsymbol{x}_{0: t-1}^{(i)}\right)}{\pi\left(\boldsymbol{x}_{t}^{(i)} \mid \boldsymbol{x}_{0: t-1}^{(i)}, \boldsymbol{y}_{1: t}\right)} .
$$

The simplest implementation consists of adopting the transition prior as the proposal distribution:

$$
\pi\left(\boldsymbol{x}_{t}^{(i)} \mid \boldsymbol{x}_{0: t-1}^{(i)}, \boldsymbol{y}_{1: t}\right)=p_{x}\left(\boldsymbol{x}_{t} \mid \boldsymbol{x}_{t-1}, u_{t}\right)
$$

in which case the weights are updated according to the likelihood function:

$$
w_{t}^{(i)} \propto w_{t-1}^{(i)} p\left(\boldsymbol{y}_{t} \mid \boldsymbol{x}_{t}^{(i)}\right) .
$$

The particle filter algorithm consists of 2 steps: (i) the sequential importance sampling step and (ii) the selection step. The selection (resampling) step replaces the weighted particles by unweighted particles in order to avoid the collapse of the Monte Carlo approximation caused by the variance increase of the weights.

\section{DIFFERENTIAL GEOMETRY TOOLS}

We devote this section to briefly introduce some concepts of Riemannian geometry. These concepts are necessary to the design of the particle filter on Riemannian manifolds (Section 4). For further details on Riemannian geometry, refer to [7].

In differential geometry, one is interested in intrinsic geometric properties which are invariant with respect to the choice of the coordinate system. This can be achieved by imposing smooth transformations between local coordinate systems (see figure 1). The following definition of differentiable manifold formalizes this concept in a global setting:

Definition 1 A differentiable (or smooth) manifold $\mathcal{M}$ is a topological manifold with a family $\mathcal{U}=\left\{U_{\alpha}, \phi_{\alpha}\right\}$ of coordinate neighborhoods such that:

1. The $U_{\alpha}$ cover $\mathcal{M}$,

2. for any $\alpha, \beta$, if the neighborhoods intersection $U_{\alpha} \cap$ $U_{\beta}$ is non empty, then $\phi_{\alpha} \circ \phi_{\beta}^{-1}$ and $\phi_{\beta} \circ \phi_{\alpha}^{-1}$ are diffeomorphisms of the open sets $\phi_{\beta}\left(U_{\alpha} \cap U_{\beta}\right)$ and $\phi_{\alpha}\left(U_{\alpha} \cap U_{\beta}\right)$ of $\mathbb{R}^{n}$,

3. any coordinate neighborhood $(V, \psi)$ meeting the property (2) with every $U_{\alpha}, \phi_{\alpha} \in \mathcal{U}$ is itself in $\mathcal{U}$

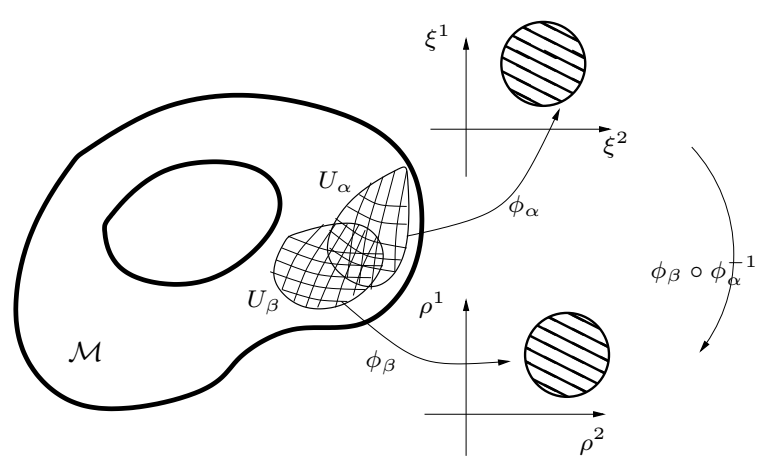

Fig. 1. Differentiable manifold

Geodesics. A geodesic between two endpoints $\gamma(a)$ and $\gamma(b)$ on a Riemannian manifold $(\mathcal{M}, g)$ endowed with a Riemannian metric $g$ is a curve $\gamma:[a, b] \longrightarrow \mathcal{M}$ which is locally defined as the shortest curve on the manifold connecting these endpoints. More formally, the definition of a geodesic is given by:

Definition 2 The parametrized curve $\gamma(t)$ is said to be a geodesic if its velocity (tangent vector) $d \gamma / d t$ is constant (parallel) along $\gamma$, that is if it satisfies the condition $(\delta / d t)(d \gamma / d t)=0$, for $a<t<b$. 
Geodesic distance. The geodesic distance $\mathcal{D}(p, q)$ between two points $p$ and $q$ on a Riemannian manifold $(\mathcal{M}, g)$ is the length of the geodesic curve $\gamma_{g}:[a, b] \longrightarrow \mathcal{M}$ connecting $p$ and $q$ :

$$
\mathcal{D}(p, q)=\left\|\gamma_{g}\right\|=\int_{a}^{b} \sqrt{g_{i j} \dot{\gamma}^{i} \dot{\gamma}^{j}} d t .
$$

The geodesic distance can also be defined as the shortest distance (over smooth curves) between two points on the manifold endowed by a Riemannian connection.

Exponential mapping. The exponential mapping is a central concept when designing filtering methods on Riemannian manifolds. In fact, it represents an interesting tool to build a bridge between an Euclidean space and the Riemannian manifold. For a point $p$ and a tangent vector $X \in$ $T_{p}(\mathcal{M})$, let $\gamma: t \Longrightarrow \gamma(t)$ be the geodesic such that $\gamma(0)=$ $p$ and $\frac{d \gamma}{d t}(0)=X$. The exponential mapping of $X$ is defined as $\mathcal{E}_{p}(X)=\gamma(1)$. In other words, the exponential mapping assigns to the tangent vector $X$ the endpoint of the geodesic whose velocity at time $t=0$ is the vector $X$ (see figure 2). It can be shown that there exist an neighborhood $U$ of 0 in $T_{p}(\mathcal{M})$ and a neighborhood $V$ of $p$ in $\mathcal{M}$ such that $\left.\mathcal{E}_{p}\right|_{U}$ is a diffeomorphism from $U$ to $V$. Also, note that since the velocity $d \gamma / d t$ is constant along the geodesic $\gamma(t)$, its length $L$ from $p$ to $\mathcal{E}_{p}(X)$ is:

$$
L=\int_{0}^{1}\left\|\frac{d \gamma}{d t}\right\| d t=\int_{0}^{1}\|X\| d t=\|X\| .
$$

The exponential mapping $\mathcal{E}_{p}(X)$ corresponds thus to the unique point on the geodesic whose distance from $p$ is the length of the vector $X$.

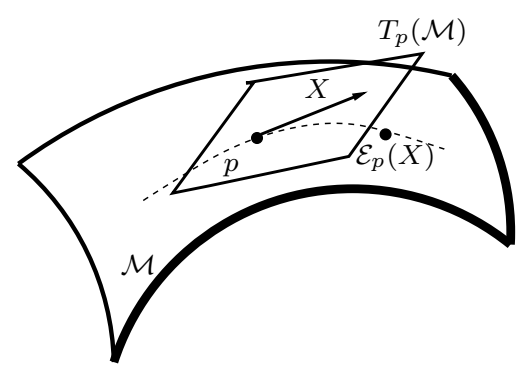

Fig. 2. Exponential mapping on the manifold

\section{PARTICLE FILTERING ON RIEMANNIAN MANIFOLDS}

\subsection{General scheme}

The aim of this section is to propose a general scheme for the extension of the particle filtering method on a Riemannian manifold. The hidden state $\boldsymbol{x}$ is constrained to lie in a Riemannian manifold $(\mathcal{M}, \boldsymbol{g}, \nabla)$ endowed with a Riemannian metric $\boldsymbol{g}$ and an affine connection $\nabla$. The system evolves according to the following nonlinear dynamics:

$$
\left\{\begin{array}{ccc}
\boldsymbol{x}_{t} & \sim p_{x}\left(\boldsymbol{x}_{t} \mid \boldsymbol{x}_{t-1}, u_{t}\right), \boldsymbol{x} \in \mathcal{M} \\
\boldsymbol{y}_{t} & \sim p_{y}\left(\boldsymbol{y}_{t} \mid \boldsymbol{x}_{t}, u_{t}\right),
\end{array}\right.
$$

where the Markov chain (random walk) $p_{x}\left(\boldsymbol{x}_{t} \mid \boldsymbol{x}_{t-1}, u_{t}\right)$ on the manifold $\mathcal{M}$ is defined according to the following generating mechanism:

1. Draw a sample $\boldsymbol{v}_{t}$ on the tangent space $\mathcal{T}_{\boldsymbol{x}_{t-1}} \mathcal{M}$ according to a pdf $p_{\boldsymbol{v}}($.$) .$

2. $\boldsymbol{x}$ is obtained by the exponential mapping of $\boldsymbol{v}_{t}$ according to the affine connection $\nabla$.

In other words, a random vector $\boldsymbol{v}_{t}$ is drawn on the tangent space $\mathcal{T}_{\boldsymbol{x}_{t-1}} \mathcal{M}$ by the usual Euclidean random technics. Then, the exponential mapping allows the transformation of this vector to a point $\boldsymbol{x}_{t}$ on the Riemannian manifold. The point $\boldsymbol{x}_{t}$ is the endpoint of the geodesic starting from $\boldsymbol{x}_{t-1}$ with a random initial velocity vector $\boldsymbol{v}_{t}$. Figure 3 illustrates the transition dynamics on a Riemannian manifold $\mathcal{M}$.

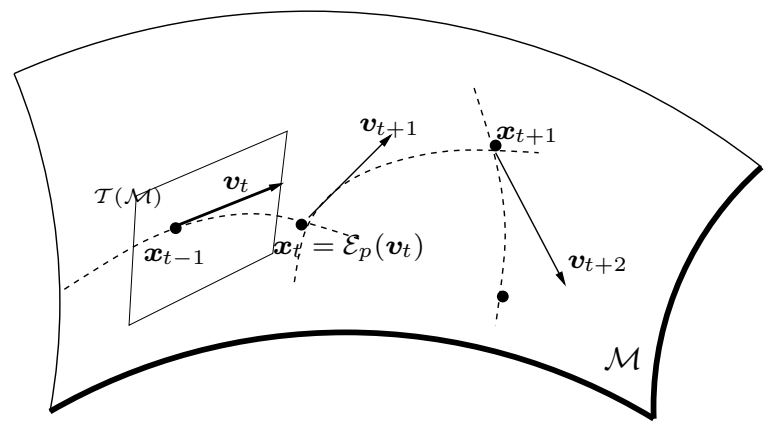

Fig. 3. Markov chain on a Riemannian manifold

As a generating stochastic mechanism is properly defined on the manifold, the particle filtering is naturally extended. It simply consists in propagating the trajectories on the manifold by the random walk process, weighting the particles by the likelihood function and sampling with replacement. The proposed general scheme is depicted in figure 1 .

\subsection{Point estimates}

Any quantity of interest $h(\boldsymbol{x})$ can be estimated by its $a$ posteriori expectation, minimizing the expected mean square error. The empirical mean of the transformed particles $h\left(\boldsymbol{x}_{t}^{(i)}\right)$ represents an unbiased Mont-Carlo estimation of the a posteriori expectation. Averaging in the manifold context is no 


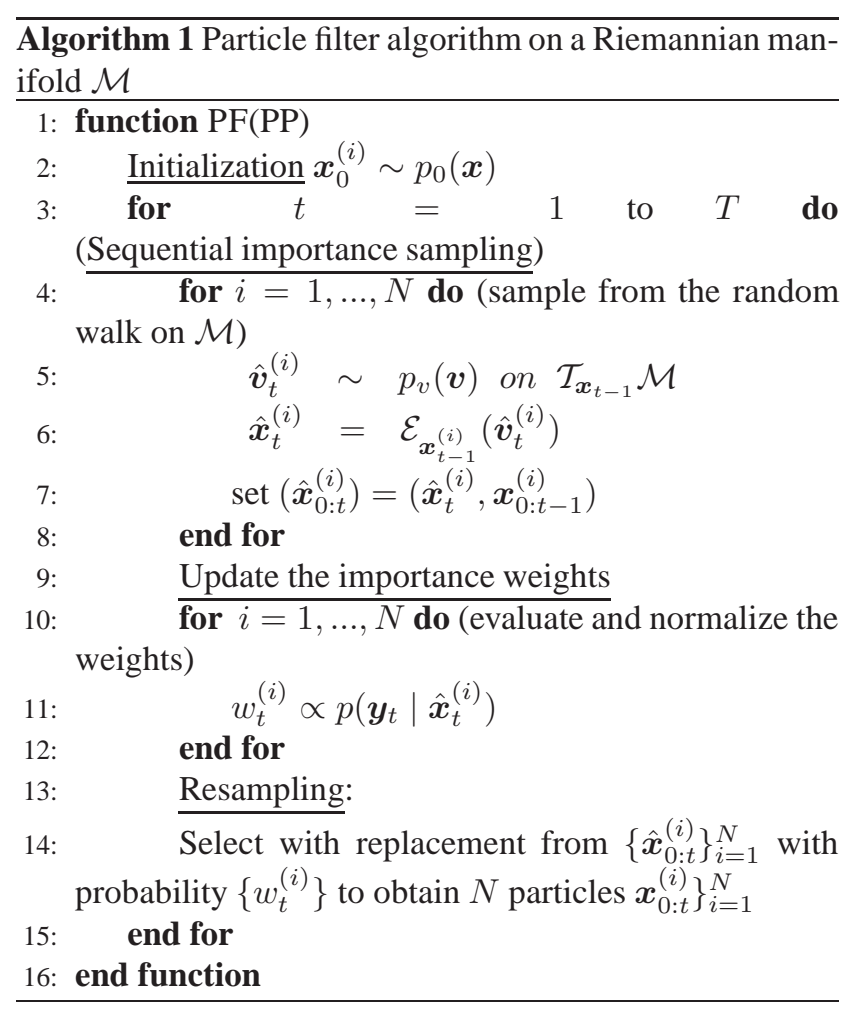

more a valid operation: The empirical mean could be located outside the manifold or the averaging itself does not have a meaning in the absence of a summation operator on the manifold. In order to obtain a valid point estimate, one should rather minimize the mean square error, where the error is evaluated by the geodesic distance $\mathcal{D}$ on the manifold (related to the connection $\nabla$ ). Following the work of Fréchet [8], the point estimate can be defined by the intrinsic mean (also called Riemannian barycenter). The intrinsic mean has the following expression:

$$
\begin{aligned}
\hat{\boldsymbol{x}}_{t} & =\arg \min _{\boldsymbol{x}_{t} \in \mathcal{M}} \mathrm{E}\left[\left(\mathcal{D}\left(\boldsymbol{x}_{t}, \boldsymbol{s}_{t}\right)\right)^{2}\right] \\
& =\arg \min _{\boldsymbol{x}_{t} \in \mathcal{M}} \int\left(\mathcal{D}\left(\boldsymbol{x}_{t}, \boldsymbol{s}_{t}\right)\right)^{2} p\left(\boldsymbol{s}_{t} \mid y_{1 . . T}\right) d_{\boldsymbol{\mu}} \boldsymbol{s}_{t}
\end{aligned}
$$

where the expectation operator is computed with respect to the a posteriori probability density $p\left(s_{t} \mid y_{1 . . T}\right)$ and a dominating measure $d_{\boldsymbol{\mu}}$.

Computation of the point estimate (5) involves an integration operation (with respect to $s_{t} \in \mathcal{M}$ and according to the posterior distribution) and a constrained optimization operation on the manifold $\mathcal{M}$. The integral can be approximated (as in the Euclidean case) by an empirical weighted sum of the geodesic distances applied on the particles which are obtained with their weights by the particle filter algorithm. The point estimate is then computed by the mini- mization of an approximated expectation expression:

$$
\hat{\boldsymbol{x}}_{t}=\underset{\boldsymbol{x}_{t} \in \mathcal{M}}{\arg \min } \sum_{i=1}^{N} w_{t}^{(i)}\left(\mathcal{D}\left(\boldsymbol{x}_{t}, \boldsymbol{s}_{t}^{(i)}\right)\right)^{2}
$$

where $\boldsymbol{s}_{t}^{(i)}$ and $w_{t}^{(i)}$ are the particles and their weights computed recursively by the particle filter algorithm.

Concerning the constrained optimization in (6), a gradient descent like algorithm can be designed on the manifold based on the exponential mapping which plays again a key role in transferring Euclidean technics to a Riemannian manifold context. Denoting by $\mathcal{J}\left(\boldsymbol{x}_{t}\right)$ the objective function to be minimized with respect to $\boldsymbol{x}_{t}$,

$$
\mathcal{J}\left(\boldsymbol{x}_{t}\right)=\sum_{i=1}^{N} w_{t}^{(i)}\left(\mathcal{D}\left(\boldsymbol{x}_{t}, \boldsymbol{s}_{t}^{(i)}\right)\right)^{2},
$$

a gradient flow $\boldsymbol{x}_{t}^{(l)}$, starting from an initial guess $\boldsymbol{x}_{t}^{(l)}$ and converging to the solution $\hat{\boldsymbol{x}}_{t}$, can be defined by moving in the direction of the opposite of the objective function derivative $\nabla \mathcal{J}\left(\boldsymbol{x}_{t}^{(l)}\right)$. As the function derivative $\nabla \mathcal{J}\left(\boldsymbol{x}_{t}^{(l)}\right)$ lies in the tangent space $\mathcal{T}_{\boldsymbol{x}_{t}^{(l)}}(\mathcal{M})$, the exponential mapping can be used to map the opposite derivative vector to the next point $\boldsymbol{x}_{t}^{(l+1)}$. The gradient-like descent algorithm is then derived as follows:

$$
\boldsymbol{x}_{t}^{(l+1)}=\mathcal{E}_{\boldsymbol{x}_{t}^{(l)}}\left(-\nabla \mathcal{J}\left(\boldsymbol{x}_{t}^{(l)}\right)\right)
$$

Figure 4 illustrates an iteration of the gradient descent algorithm on a Riemannian manifold.

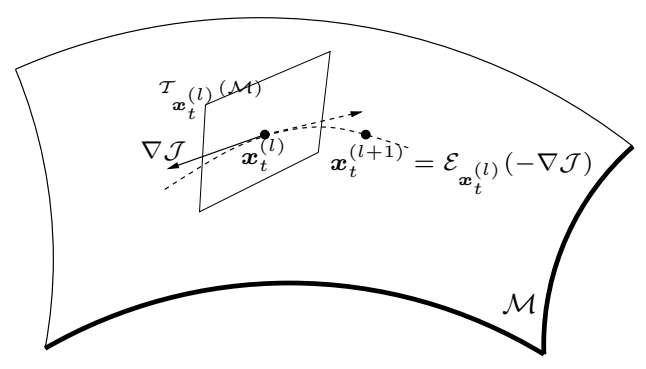

Fig. 4. A gradient descent step on a Riemannian manifold

\section{APPLICATION TO TRACKING WITH UNKNOWN TIME-VARYING NOISE COVARIANCE}

\subsection{General algorithm}

In classical filtering methods applied on state-space models, it is usually assumed that the noise statistics are known. In other words, given the hidden state $\boldsymbol{x}_{t}$, the likelihood function $p\left(\boldsymbol{y}_{t} \mid \boldsymbol{x}_{t}\right)$ can be exactly computed. However, in real 
situations, the noise is generally related to the sensor imperfections which may be varying and unknown. For instance, assuming (for simplicity) an additive Gaussian noise, the variation of the noise covariance $\boldsymbol{\Sigma}_{t}$ is related to the degradation of the sensing system. Fixing a constant value for the noise covariance may lead to poor tracking performances. An optimal procedure to deal with the unknown time-varying covariance matrix is to design a Bayesian filter to jointly track the hidden state of interest $\boldsymbol{x}_{t}$ and the noise covariance $\boldsymbol{\Sigma}_{t}$. In this section, we consider the tracking of a mobile target. The hidden state $\boldsymbol{x}_{t}$ contains the position and the velocity of the target. It belongs thus to the Euclidean space $\mathbb{R}^{4}$. However, the noise covariance matrix lies in the Riemannian manifold of positive definite matrices $\mathcal{S}_{+}$. The general scheme defined in the previous Section 4 should be applied in order to track the target and to online estimate the noise covariance.

The hidden state $\boldsymbol{x}_{t}$ is assumed to follow a general parametric prior model $p\left(\boldsymbol{x}_{t} \mid \boldsymbol{x}_{t-1}, \boldsymbol{\alpha}\right)$, where the parameters $\boldsymbol{\alpha}$ are assumed to be known. In order to describe the temporal correlation of the noise covariance trajectory on the Riemannian manifold of positive definite matrices $\mathcal{S}_{+}$, we define the Generalized Gaussian random walk $\boldsymbol{\Sigma}_{t} \sim$ $\mathcal{G N}\left(\boldsymbol{\Sigma}_{t} \mid \boldsymbol{\Sigma}_{t-1}, \boldsymbol{\Lambda}\right)$ as follows:

1. Sample a Gaussian symmetric velocity matrix $B \in$ $\mathcal{S}$ with a precision $\Lambda\left(\frac{n_{x}\left(n_{x}+1\right)}{2} \times \frac{n_{x}\left(n_{x}+1\right)}{2}\right.$ matrix $)$ : $\boldsymbol{B} \sim \mathcal{N}(0 ; \boldsymbol{\Lambda})$

2. The next matrix $\boldsymbol{\Sigma}_{t}$ is then obtained by:

$$
\boldsymbol{\Sigma}_{t}=\mathcal{E}_{\boldsymbol{\Sigma}_{t-1}}(\boldsymbol{B})=\boldsymbol{\Sigma}_{t-1}^{1 / 2} \exp \left[\boldsymbol{\Sigma}_{t-1}^{-1 / 2} \boldsymbol{B} \boldsymbol{\Sigma}_{t-1}^{-1 / 2}\right] \boldsymbol{\Sigma}_{t-1}^{1 / 2}
$$

The state-space model is then described by the following equations,

$$
\left\{\begin{aligned}
\boldsymbol{x}_{t} & \sim p_{x}\left(\boldsymbol{x}_{t} \mid \boldsymbol{x}_{t-1}, \boldsymbol{\alpha}\right) \\
\boldsymbol{\Sigma}_{t} & \sim \mathcal{G N}\left(\boldsymbol{\Sigma}_{t} \mid \boldsymbol{\Sigma}_{t-1}, \boldsymbol{\Lambda}\right) \\
\boldsymbol{y}_{t} & \sim \mathcal{N}\left(\boldsymbol{y}_{t} \mid f\left(\boldsymbol{x}_{t}\right), \boldsymbol{\Sigma}_{t}\right),
\end{aligned}\right.
$$

where $f($.$) is the sensing function and \mathcal{N}($.$) stands for the$ Gaussian distribution. Contrary to the usual assumption of a constant known covariance, the case of a stochastic varying noise covariance represents an elegant way to deal with temporal degradation of the sensing system.

The proposed particle filter jointly estimates the hidden target position $\boldsymbol{x}_{t}$ (belonging to an Euclidean space) and the noise covariance $\boldsymbol{\Sigma}_{t}$ (belonging to the Riemannian manifold $\mathcal{S}_{+}$) as follows,

1. Propagate the trajectories $\left(\boldsymbol{\Sigma}_{0: t-1}^{(i)}, \boldsymbol{x}_{0: t-1}^{(i)}\right)$ by generating the samples $\left(\boldsymbol{\Sigma}_{t}^{(i)}, \boldsymbol{x}_{t}^{(i)}\right)$ according to the prior models $\mathcal{G N}\left(\boldsymbol{\Sigma}_{t} \mid \boldsymbol{\Sigma}_{t-1}, \boldsymbol{\Lambda}\right)$ and $p\left(\boldsymbol{x}_{t} \mid \boldsymbol{x}_{t-1}, \boldsymbol{\alpha}\right)$.
2. Update the importance weights which are proportional to the likelihood function $\mathcal{N}\left(\boldsymbol{y}_{t} \mid f\left(\boldsymbol{x}_{t}\right), \boldsymbol{\Sigma}_{t}\right)$.

The explicit solution of the geodesic distance allows also the implementation of the intrinsic mean for the tracking of the covariance matrix. In fact, approximating the expected error by the empirical weighted sum of geodesic distances, the point estimate is defined as follows:

$$
\begin{aligned}
\overline{\boldsymbol{\Sigma}}_{t} & =\arg \min _{\boldsymbol{\Sigma} \in \mathcal{S}_{+}} \sum_{i=1}^{N} w_{t}^{(i)}\left(\mathcal{D}\left(\boldsymbol{\Sigma}, \boldsymbol{\Sigma}_{t}^{(i)}\right)\right)^{2} \\
& =\arg \min _{\boldsymbol{\Sigma} \in \mathcal{S}_{+}} \sum_{i=1}^{N} w_{t}^{(i)} \frac{1}{2} \operatorname{tr} L n^{2}\left(\boldsymbol{\Sigma}^{-1 / 2} \boldsymbol{\Sigma}_{t}^{(i)} \boldsymbol{\Sigma}^{-1 / 2}\right)
\end{aligned}
$$

The gradient of the objective function, belonging to the tangent space $\mathcal{T}_{\Sigma} \mathcal{S}_{+}$, has the following expression:

$$
\nabla \mathcal{J}(\boldsymbol{\Sigma})=\frac{\boldsymbol{\Sigma}}{N} \sum_{i=1}^{N} \operatorname{Ln}\left(\left(\boldsymbol{\Sigma}_{t}^{(i)}\right)^{-1} \boldsymbol{\Sigma}\right)
$$

Given the explicit expression of both the gradient (9) and the exponential mapping on the manifold $\mathcal{S}_{+}$, the gradientdescent algorithm scheme for the computation of $\overline{\boldsymbol{\Sigma}}_{t}$ is efficiently implemented. Given an initial guess $\overline{\boldsymbol{\Sigma}}^{(0)}$, a gradient flow $\overline{\boldsymbol{\Sigma}}^{(l)}$ evolving towards the solution is defined as follows:

$$
\begin{aligned}
\overline{\mathbf{\Sigma}}^{(l+1)} & =\mathcal{E}_{\overline{\boldsymbol{\Sigma}}^{(l)}}\left(-\nabla \mathcal{J}\left(\overline{\boldsymbol{\Sigma}}^{(l)}\right)\right) \\
& =\left(\overline{\boldsymbol{\Sigma}}^{(l)}\right)^{1 / 2} \exp \left[\left(-\overline{\boldsymbol{\Sigma}}^{(l)}\right)^{-1 / 2} \nabla \mathcal{J}\left(\overline{\boldsymbol{\Sigma}}^{(l)}\right)^{-1 / 2}\right]\left(\overline{\boldsymbol{\Sigma}}^{(l)}\right)^{1 / 2}
\end{aligned}
$$

\subsection{Simulation results}

We consider the tracking of a target moving over a 2-D field. The state $\boldsymbol{x}_{t}=\left[\boldsymbol{x}_{t}^{p}, \boldsymbol{x}_{t}^{v}\right]$ is formed by the position and the velocity of the target. For simplicity, we assume a kinematic parametric model for the transition dynamics of the hidden state:

$$
\begin{array}{r}
\left(\begin{array}{c}
\boldsymbol{x}_{t}^{p} \\
\boldsymbol{x}_{t}^{v}
\end{array}\right)=\left(\begin{array}{cccc}
1 & 0 & T_{s} & 0 \\
0 & 1 & 0 & T_{s} \\
0 & 0 & 1 & 0 \\
0 & 0 & 0 & 0
\end{array}\right)\left(\begin{array}{c}
\boldsymbol{x}_{t-1}^{p} \\
\boldsymbol{x}_{t-1}^{v}
\end{array}\right) \\
+\left(\begin{array}{cc}
T_{s}^{2} / 2 & 0 \\
0 & T_{s}^{2} / 2 \\
T_{s} & 0 \\
0 & T_{s}
\end{array}\right) \boldsymbol{u}_{t}
\end{array}
$$

where the sampling interval is $T_{s}=0.1 s$ and $\boldsymbol{u}_{t}$ is a zeromean white Gaussian noise.

The observations are obtained through a network of 400 range-bearing sensors deployed randomly in the field under surveillance. At each time $t$, a selected node (according to 
the proximity to the target) obtains an observation of the target position through a range-bearing model:

$$
\left(\begin{array}{c}
y_{t}^{r} \\
y_{t}^{\theta}
\end{array}\right)=\left(\begin{array}{c}
\frac{p}{\left\|\boldsymbol{s}_{m}-\boldsymbol{x}_{t}\right\|+0.5} \\
\arctan \frac{s_{2}-x_{2}}{s_{1}-x_{1}}
\end{array}\right)+\boldsymbol{\Sigma}_{t} \boldsymbol{v}_{t}
$$

where $\boldsymbol{s}_{m}=\left(s_{1}, s_{2}\right)$ and $\boldsymbol{x}_{t}=\left(x_{1}, x_{2}\right)$ are the node and the target positions at time $t, p$ (set to 10) is the energy emitted by the target (measured at a reference distance of 1 meter) and $\boldsymbol{v}_{t}$ is a white Gaussian random vector. The corrupting noise has a covariance $\boldsymbol{\Sigma}_{t}$ evolving in time as depicted in figure 6: constant for the first $T / 4 s$, increasing with a linear slope for $T / 2 s$ and constant for the last $T / 4 s$.

The particle filter is applied to jointly estimate the target position and the noise covariance matrix. Figure 5 illustrates the target tracking performances. The trajectory of the target is recovered with a mean square error of $0.39 \mathrm{~m}$. Figure 6 illustrates the performance of the algorithm to online track the covariance variation over time. Note that, despite their fluctuation, the estimated covariance elements follow the tendency of the true covariance elements. The fluctuation of the estimated noise covariance is mainly due to the fact that the data are less informative with respect to the covariance matrix. In fact, unlike the target position estimation, the online estimation of the covariance $\boldsymbol{\Sigma}_{t}$ is an illposed problem based on only one observation $\boldsymbol{y}_{t}$. The success of the algorithm to approximately recover the tendency of the covariance matrix is due to the Markov prior regularization defined by the Generalized Gaussian random walk $\mathcal{G N}\left(\boldsymbol{\Sigma}_{t} \mid \boldsymbol{\Sigma}_{t-1}, \boldsymbol{\Lambda}\right)$ defined in the previous subsection.

\section{CONCLUSION}

A differential-geometric framework is proposed to implement the particle filtering algorithm on Riemannian manifold. The exponential mapping plays a central role in connecting the manifold-valued particles to the samples generated on the tangent space by the usual random generating techniques on Euclidean spaces. The proposed algorithm has been applied to jointly track the target position with the time-varying noise covariance.

\section{REFERENCES}

[1] A. Doucet, S. Godsill, and C. Andrieu, "On sequential Monte Carlo sampling methods for Bayesian filtering," Statistics and Computing, vol. 10, no. 3, pp. 197-208, 2000.

[2] X. Liu, A. Srivastava, and K. Gallivan, "Optimal linear representations of images for object recognition," IEEE Pattern Analysis and Machine Intelligence, vol. 25, no. 5, pp. 662666, May 2004.

[3] C. Lenglet, M. Rousson, R. Deriche, and O. Faugeras, "Statistics on the manifold of multivariate normal distributions: Theory and application to diffusion tensor MRI processing,",

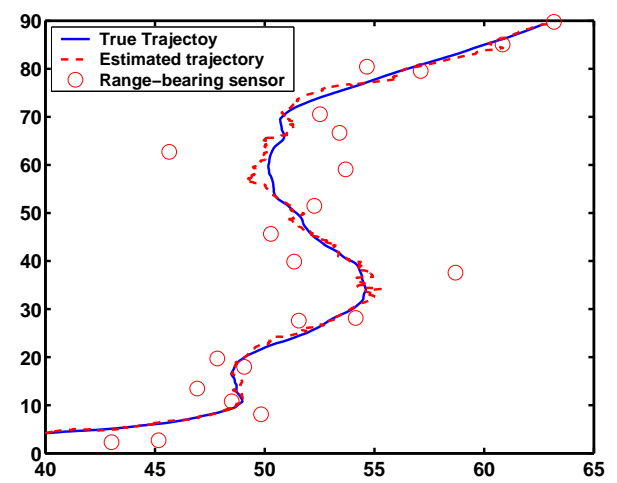

Fig. 5. Target tracking with unknown noise covariance.
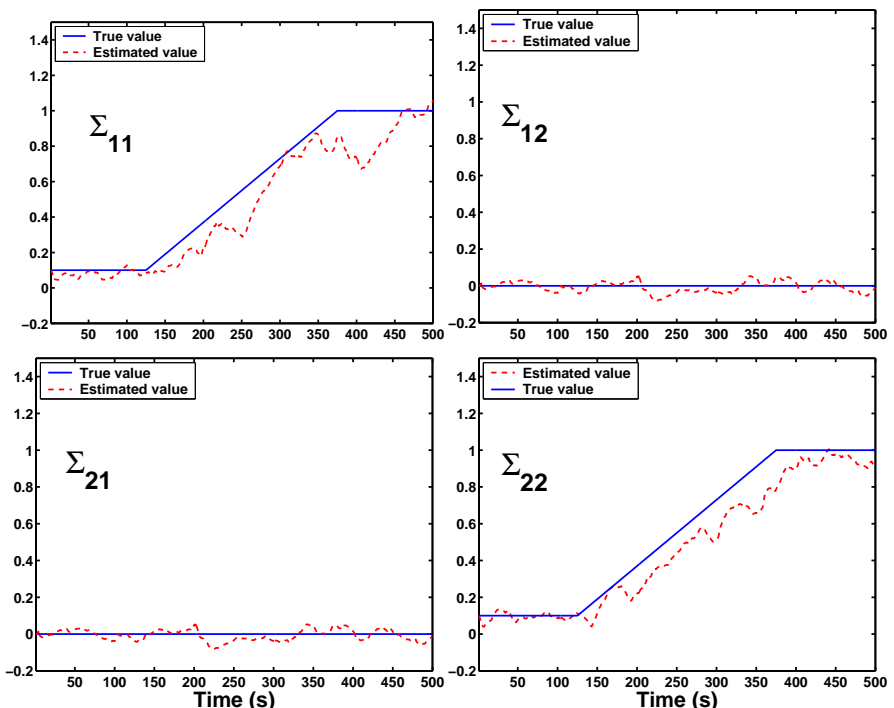

Fig. 6. Online estimation of the noise covariance elements.

Journal of Mathematical Imaging and Vision, vol. 25, pp. 423-444, October 2006.

[4] S. Fiori, "Geodesic-based and projection-based neural blind deconvolution algorithms," Signal processing, vol. 88, pp. 521-538, 2008.

[5] A. Srivastava and E. Klassen, "Bayesian and geometric subspace tracking," Advances in Applied Probability, vol. 36, no. 1, pp. 43-56, March 2004.

[6] Hichem Snoussi and Ali Mohammad-Djafari, "Particle Filtering on Riemannian Manifolds," in Bayesian Inference and Maximum Entropy Methods, Ali Mohammad-Djafari, Ed. MaxEnt Workshops, July 2006, pp. 219-226, Amer. Inst. Physics.

[7] W.M. Boothby, An Introduction to Differential Manifolds and Riemannian Geometry, Academic Press, Inv., 1986.

[8] M. Fréchet, "Les éléments aléatoires de nature quelconque dans un espace distancié," Ann. Inst. H. Poincaré, 1948. 